\title{
uc.338 targets p21 and cyclin D1 via PI3K/AKT pathway activation to promote cell proliferation in colorectal cancer
}

\author{
YUE ZHANG $^{1,2^{*}}$, SHIJIA WANG ${ }^{1,2^{*}}$, WENWEI QIAN ${ }^{1,2^{*}}$, DONGJIAN JI ${ }^{1,2}$, QINGYUAN WANG ${ }^{1,2}$, \\ ZHIYUAN ZHANG ${ }^{1,2}$, SEN WANG ${ }^{1,2}$, BING JI ${ }^{1,2}$, ZAN FU $^{2}$ and YUEMING SUN ${ }^{2}$ \\ ${ }^{1}$ The First School of Clinical Medicine, Nanjing Medical University; ${ }^{2}$ Department of General Surgery, \\ The First Affiliated Hospital of Nanjing Medical University, Nanjing, Jiangsu 210029, P.R. China
}

Received January 10, 2018; Accepted May 31, 2018

DOI: $10.3892 / o r .2018 .6480$

\begin{abstract}
Ultraconserved regions (UCRs) are 481 segments that have been strictly conserved among many mammalian species for hundreds of years. Their transcribed products belong to long non-coding RNAs and are closely involved in the progression of various tumors. uc. 338 has been reported to promote cell proliferation in hepatocellular and lung cancers. However, the role of uc.338 in colorectal cancer (CRC) proliferation remains unclear. In the present study, we first detected the expression of uc.338 in human tissues and analyzed the relationship between uc.338 expression and clinical features of CRC. We then investigated the biological function of uc.338 in CRC proliferation in vitro and in vivo. Finally, we explored the potential mechanism by which uc.338 promotes the proliferation of CRC cells. Our results indicated that uc.338 was upregulated in CRC tissues and higher uc.338 expression was associated with a larger tumor size, deeper invasion, increased lymph node metastasis and poorer prognosis. Further investigations in vivo and in vitro revealed that uc. 338 could promote proliferation and cell cycle G1/S transition, and might target p21 downregulation and cyclin D1 upregulation via the $\mathrm{PI} 3 \mathrm{~K} / \mathrm{AKT}$ pathway in CRC cells. Thus, our findings suggested
\end{abstract}

Correspondence to: Dr Zan Fu or Dr Yueming Sun, Department of General Surgery, The First Affiliated Hospital of Nanjing Medical University, 300 Guangzhou Road, Nanjing, Jiangsu 210029, P.R. China

E-mail: fuzan1971@njmu.edu.cn

E-mail: jssym@vip.sina.com

*Contributed equally

Abbreviations: CRC, colorectal cancer; UCRs, ultraconserved regions; T-UCRs, transcribed ultraconserved regions; NCCN, National Comprehensive Cancer Network; DMEM, Dulbecco's modified Eagle's medium; PBS, phosphate-buffered saline; qPCR, quantitative real-time PCR; siRNA, small interfering RNA; CCK-8, Cell Counting Kit-8; EdU, 5-ethynyl-2'-deoxyuridine

Key words: ultraconserved region, uc.338, p21, cyclin D1, cell proliferation, PI3K, AKT, colorectal cancer that uc.338 acts as an oncogene by promoting proliferation in CRC cells, and could become a novel target for CRC detection and therapy.

\section{Introduction}

Colorectal cancer (CRC) remains a critical health concern worldwide, with an estimated 1.4 million cases occurring in 2012 (1). It has become the fourth most commonly diagnosed cancer and is the fifth leading cause of cancer-related death in China (2). Although much progress has been made, the biological mechanism and potential biomarkers of CRC remain to be clarified. Therefore, more studies on the molecular mechanism of CRC are required to facilitate the early diagnosis and treatment of CRC.

Ultraconserved regions (UCRs) are 481 DNA segments of more than $200 \mathrm{bp}$ in length that are absolutely conserved $(100 \%)$ in the human, rat and mouse genomes, most of which are also 95-99\% conserved in chickens and dogs. They are always classified into three types, namely, exonic, non-exonic, and possibly exonic (3). Most UCRs can be transcribed and their transcriptional products, called T-UCRs, represent an important category of long non-coding RNAs (lncRNAs) (4). T-UCRs have been reported to be involved in various cancer processes (5-9). As a member of the T-UCR family, uc.338 has been reported to promote tumorigenesis in hepatocellular carcinoma (9-11), cervical cancer (12) and lung carcinoma (13). In CRC, uc.338 was reported to promote the invasion and metastasis of HCT116 and SW480 cells (14). However, the exact role of uc.338 in CRC proliferation remains unknown.

Therefore, in the present study, we detected the role and potential mechanism of uc.338 in CRC proliferation. Our results indicated that uc.338 upregulation is vital for the progression of CRC, highlighting the potential of uc.338 as a therapeutic target in CRC.

\section{Materials and methods}

Human tissues. Our study was approved by the Ethics Committee of the First Affiliated Hospital of Nanjing Medical University. From July 2010 to December 2012, we collected 100 pairs of human CRC tissues and adjacent normal tissues 
from patients after they had signed an informed consent form. Of the patients, 64 were males and 36 were females. Ages of patients at the time of surgery ranged from 27 to 82 years (60.14 \pm 11.72 years). The tumor tissues and adjacent normal tissues were collected in liquid nitrogen within $5 \mathrm{~min}$, and then immediately transferred to a $-70^{\circ} \mathrm{C}$ freezer for long-term storage. The tumor-node-metastasis (TNM) stage was classified according to the National Comprehensive Cancer Network (NCCN). In this study, none of the patients received any preoperative treatments.

Cell lines and cell culture. CRC cell lines LoVo, HCT116, DLD-1, and human colon epithelial mucosal cell line NCM460 were maintained in our laboratory. All cell lines were cultured with Dulbecco's modified Eagle's medium (DMEM; Winsent, Quebec, Canada) supplemented with $10 \%$ fetal bovine serum (Winsent), $100 \mathrm{U} / \mathrm{ml}$ penicillin, and $100 \mu \mathrm{g} / \mathrm{ml}$ streptomycin in a moist incubator (stabilized at $5 \% \mathrm{CO}_{2}$ and $\left.37^{\circ} \mathrm{C}\right)$.

Quantitative real-time PCR ( $q P C R)$. Total RNA was extracted from CRC tissues and adjacent normal tissues using Invitrogen ${ }^{\mathrm{TM}}$ TRIzol reagent (Thermo Fisher Scientific, Inc., Waltham, MA, USA) according to the manufacturer's instructions. The RNA was converted to cDNA using the PrimeScript RT reagent kit (Takara Biotechnology, Co., Ltd., Dalian, China). The qPCR experiment was performed using a SYBR Green PCR kit (Roche Diagnostics, Indianapolis, IN, USA) in a volume of $20 \mu \mathrm{l}$, and then the final reaction was carried using athe Applied Biosystems Step-One Plus Real-Time PCR System (Thermo Fisher Scientific, Inc.). The qPCR cycling was performed as follows: Hot-start DNA polymerase activation at $95^{\circ} \mathrm{C}$ for $10 \mathrm{~min} ; 40$ cycles of $95^{\circ} \mathrm{C}$ for $15 \mathrm{sec}$, and $60^{\circ} \mathrm{C}$ for $1 \mathrm{~min}$; followed by 1 cycle of melting curve analysis of $95^{\circ} \mathrm{C}$ for $15 \mathrm{sec}, 60^{\circ} \mathrm{C}$ for $1 \mathrm{~min}$, and $95^{\circ} \mathrm{C}$ for $15 \mathrm{sec}$. The primer sequences for uc.338 were as follows: 5'-AGCGACAGTGCGAGCTTT-3' (forward) and 5'-TTCCGA GTGAGTTAGGAAGG-3' (reverse). The primer sequences for glyceraldehyde-3-phosphate dehydrogenase $(G A P D H)$ were as follows: 5'-GTGGACATCCGCAAAGAC-3' (forward) and 5'-AAAGGGTGTAACGCAACTA-3' (reverse).

siRNA and plasmid transfection. Small interfering RNA (siRNA) targeting uc.338 and a negative control (NC) siRNA were designed by Genepharma Corp. (Shanghai, China) and transferred into LoVo and HCT116 cells. The target sequence was as follows: siuc. 338, 5'-CCACAGGACAGGUACAGCA-3'. For uc.338 overexpression, $5 \mu \mathrm{g}$ per-well (6-well plate) of the plasmids expressing uc. 338 and negative control sequence designed by GeneCopoeia, Inc. (Rockville, MD, USA) were transferred into DLD-1 cells. All the above siRNA sequences and plasmids were transfected using Invitrogen ${ }^{\mathrm{TM}}$ Lipofectamine 3000 (Thermo Fisher Scientific, Inc.) according to the manufacturer's protocols. Transfection efficiency was analyzed after $48 \mathrm{~h}$. Additionally, a lentivirus expressing a small hairpin RNA against uc.338 (shuc.338) for an in vivo study was designed according to the sequence of siuc.338. The phosphatidylinositol-4,5-bisphosphate 3-kinase (PI3K) inhibitor LY294002 was purchased from Cell Signaling Technology (Danvers, MA, USA).
Table I. Expression of uc.338 in colorectal carcinoma and adjacent normal tissues.

\begin{tabular}{|c|c|c|c|c|}
\hline \multirow[b]{2}{*}{ Clinical features } & \multicolumn{4}{|c|}{ uc.338 expression } \\
\hline & $\mathrm{N}$ & High & Low & P-value \\
\hline Sex & & 50 & 50 & 0.096 \\
\hline Male & 64 & 36 & 28 & \\
\hline Female & 36 & 14 & 22 & \\
\hline Age (year) & & & & 0.288 \\
\hline$>60$ & 67 & 31 & 36 & \\
\hline$\leq 60$ & 33 & 19 & 14 & \\
\hline Tumor size $(\mathrm{cm})$ & & & & $0.002^{\mathrm{a}}$ \\
\hline$>5$ & 41 & 28 & 13 & \\
\hline$\leq 5$ & 59 & 22 & 37 & \\
\hline Depth of invasion & & & & $0.032^{\mathrm{a}}$ \\
\hline $\mathrm{T} 1 / \mathrm{T} 2$ & 32 & 11 & 21 & \\
\hline T3/T4 & 68 & 39 & 29 & \\
\hline Lymph node metastasis & & & & $0.005^{\mathrm{a}}$ \\
\hline Absent & 42 & 14 & 28 & \\
\hline Present & 58 & 36 & 22 & \\
\hline Tumor stage & & & & 0.539 \\
\hline $\mathrm{I} / \mathrm{II}$ & 39 & 18 & 21 & \\
\hline III/IV & 61 & 32 & 29 & \\
\hline Liver metastasis & & & & 0.275 \\
\hline Yes & 16 & 10 & 6 & \\
\hline No & 84 & 40 & 44 & \\
\hline Location & & & & 0.546 \\
\hline Rectal & 55 & 26 & 29 & \\
\hline Colon & 45 & 24 & 21 & \\
\hline CEA (ng/ml) & & & & 0.155 \\
\hline$>4.7$ & 41 & 17 & 24 & \\
\hline$\leq 4.7$ & 59 & 33 & 26 & \\
\hline
\end{tabular}

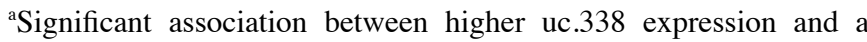
larger tumor size, deeper tumor invasion, and increased lymph node metastasis was demonstrated. CEA, carcinoembryonic antigen.

Cell proliferation assay. Cell proliferation was measured using a Cell Counting Kit-8 assay (CCK-8; Dojindo Laboratories, Tokyo, Japan) according to the manufacturer's protocol. After 24, 48, 72 and $96 \mathrm{~h}, 10 \mu$ l CCK-8 assay solution mixed with $90 \mu \mathrm{l}$ of serum-free medium was added in each well containing the cells to be tested. The absorbance was measured $2 \mathrm{~h}$ later using a microplate reader at a test wavelength of $450 \mathrm{~nm}$ and a reference wavelength of $630 \mathrm{~nm}$.

5-Ethynyl-2'-deoxyuridine (EdU) assay. The EdU assay kit (RiboBio, China) was used to detect the number of cells that were in the DNA replication period, which could indirectly reveal the cell proliferation rate. The steps were as follows. Before the addition of $\operatorname{EdU}(50 \mu \mathrm{M})$, the cells were cultured with DMEM (10\% FBS) for $24 \mathrm{~h}$ in 24 -well plates $\left(2 \times 10^{4}\right.$ cells/well). The cells were then incubated for $2 \mathrm{~h}$ at 
$\mathbf{A}_{\infty}$

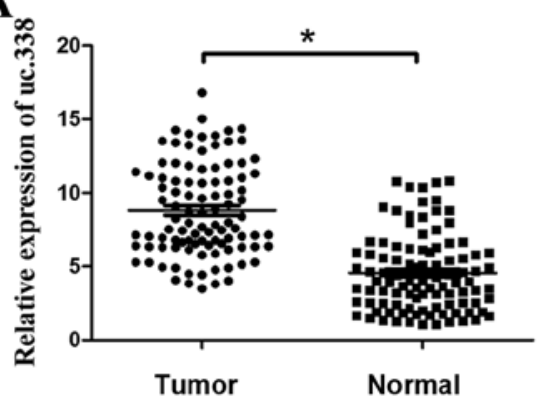

B

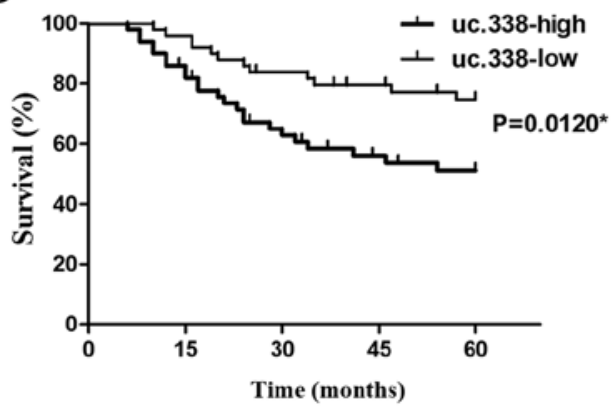

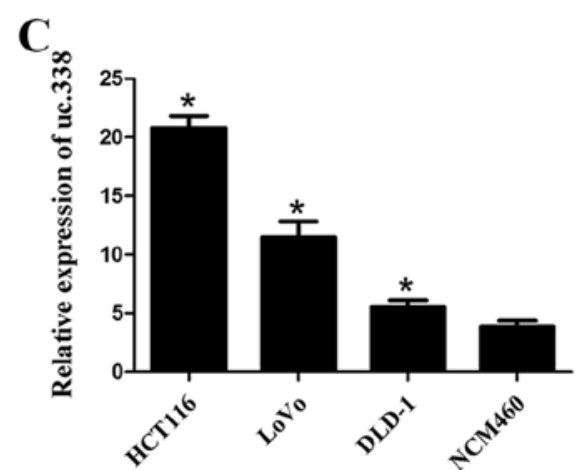

D

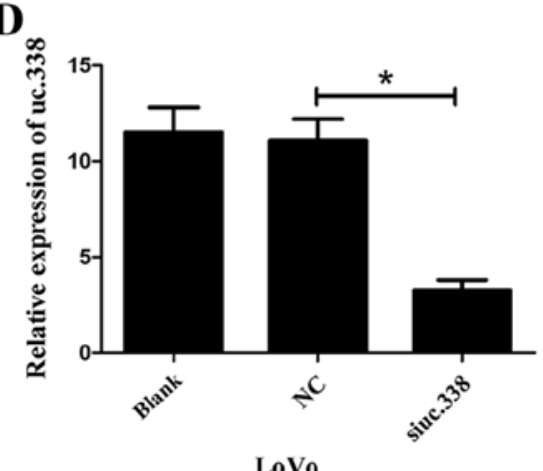

E

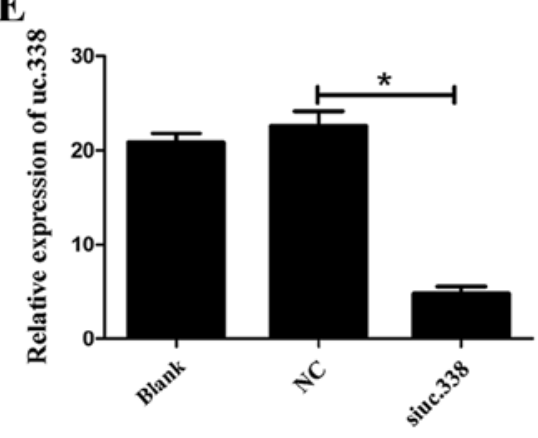

HCT116

Figure 1. mRNA expression of uc.338 in CRC tissues and CRC cells. (A) Relative mRNA expression of uc.338 in 100 pairs of CRC tissues and adjacent normal tissues detected by qPCR. (B) Kaplan-Meier survival analysis of 100 CRC patients based on uc.338 expression. (C) Relative mRNA expression of uc.338 in CRC cells and normal colon epithelial mucosa cell line (NCM460). (D and E) Relative mRNA expression of uc.338 in LoVo and HCT116 cells by siuc.338 transfection. ${ }^{*} \mathrm{P}<0.05$; data are expressed as the mean $\pm \mathrm{SD}$. CRC, colorectal cancer.

$37^{\circ} \mathrm{C}$, fixed in $4 \%$ formaldehyde for $30 \mathrm{~min}$, and permeabilized with $0.5 \%$ Triton X-100 for $10 \mathrm{~min}$ at room temperature. After washing with phosphate-buffered saline (PBS) for $5 \mathrm{~min}$, $400 \mu \mathrm{l}$ of $1 \mathrm{X}$ ApolloR reaction cocktail was added to react with the EdU for $30 \mathrm{~min}$. Finally, $400 \mu \mathrm{l}$ of Hoechst 33342 was added for $30 \mathrm{~min}$ to visualize the cell nuclei. The cells were then observed under a Nikon microscope (Nikon TI-DH; Nikon, Tokyo, Japan).

Plate colony formation assay. Five hundred cells were treated with siuc.338 and NC and cultured in wells of a 6-well plate to perform the colony formation assay. All cells were cultured with DMEM $+10 \%$ serum $+100 \mathrm{U} / \mathrm{ml}$ penicillin and $100 \mu \mathrm{g} / \mathrm{ml}$ streptomycin under the same conditions. One week later, the cells were washed with cold PBS three times at room temperature. The cells were immediately fixed with ethyl alcohol for $30 \mathrm{sec}$, and stained with crystal violet dye for $20 \mathrm{~min}$. Cell colonies ( $\geq 50$ cells/colony) were observed and imaged using a digital camera (Canon DS126211; Canon, Tokyo, Japan) in each plate after washing with PBS.

Cell cycle analysis. All the CRC cells were collected and saved in $75 \%$ ethyl alcohol at $4^{\circ} \mathrm{C}$ overnight. Before detection, the treated cells were fixed with $500 \mu \mathrm{l}$ of propidium iodide (PI) staining solution and incubated for $30 \mathrm{~min}$ in the dark. The analysis of cell cycles was performed using a fluorescence-activated cell sorting (FACS) Calibur flow cytometer with BD CellQuest software (version 3.0; BD Biosciences, Franklin Lakes, NJ, USA).
In vivo tumor xenograft model. Our animal experiments were approved by the Animal Ethics Committee of Nanjing medical university (NJMU). Fifteen male BALB/c nude mice (aged 3-4 weeks, 13-15 g weight) were purchased from the Animal Center of NJMU and were randomly divided to two groups (the LoVo cell group and the HCT116 cell group). Then, $2 \times 10^{6}$ of CRC cells mixed with $200 \mu \mathrm{l}$ PBS were subcutaneously injected into the anesthetized mice. Each mouse was randomly injected with cells treated in two different ways (shuc.338 and NC) in their right and left armpits. The mice were maintained under the following conditions: room temperature, $20-26^{\circ} \mathrm{C}$; relative humidity, 40-70\%; light/dark cycle, $12 \mathrm{~h}$; food and water, $5 \mathrm{~g}$ food and $100 \mathrm{ml}$ water per $100 \mathrm{~g}$ body weight per day. Four weeks after injection, all mice were sacrificed by cervical dislocation and all the tumors were surgically removed. The maximum tumor size was as allowable by IACUC guidelines (diameter, $1.5 \mathrm{~cm}$; area, $1.8 \mathrm{~cm}^{2}$; and volume $1.8 \mathrm{~cm}^{3}$ ).

Western blotting. Protein was extracted from CRC cells by using a Radioimmunoprecipitation assay (RIPA) kit (Beyotime Institute of Biotechnology, Shanghai, China) according to the manufacturer's protocols. The concentration was determined using the Bicinchoninic Acid Protein Assay kit (BCA). Proteins (40 $\mu \mathrm{g})$ with different molecular weights were separated on $10 \%$ SDS-PAGE gels in running buffer and transferred to polyvinylidene fluoride (PVDF) membranes (Millipore, Bedford, MA, USA) in transfer buffer. The membranes were then blocked in 5\% non-fat milk at room temperature for over $2 \mathrm{~h}$ and incubated in specific primary 
A

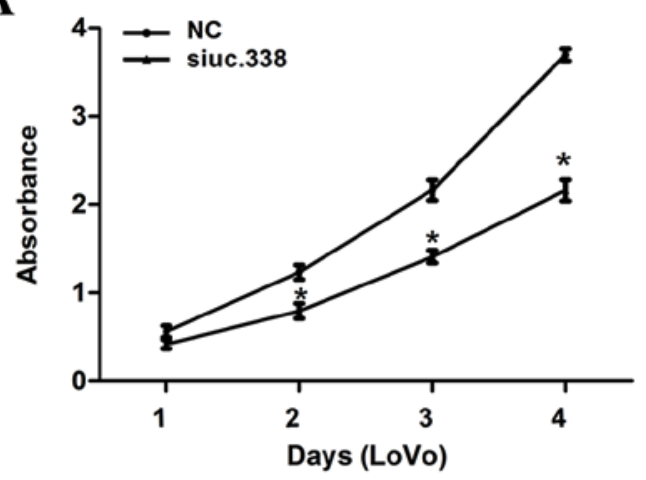

B

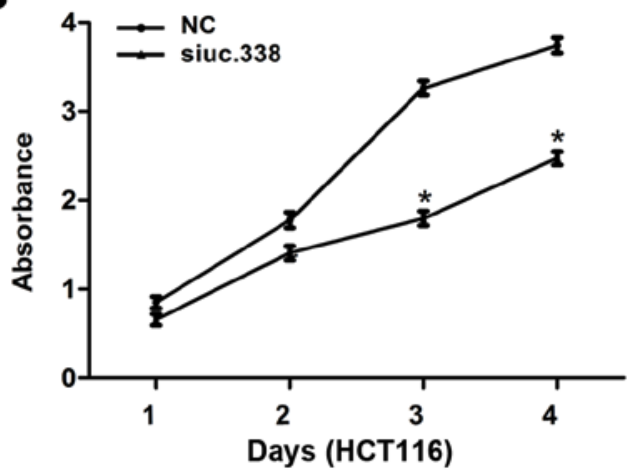

C
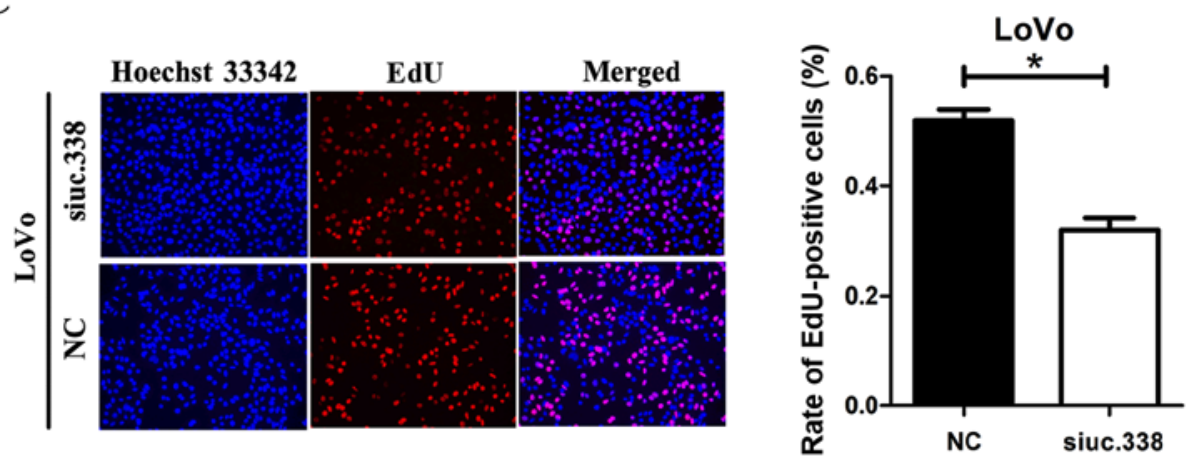

D
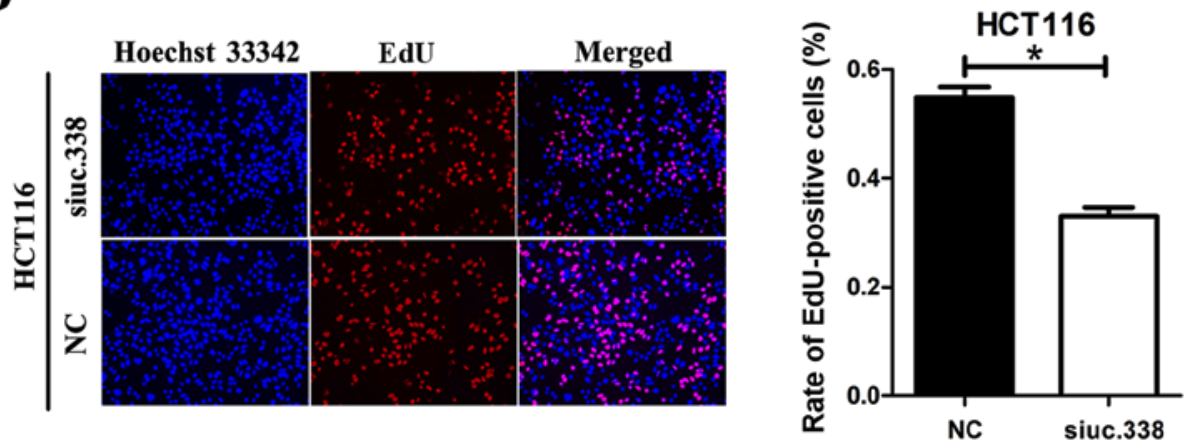

Figure 2. uc.338 promotes cell proliferation in LoVo and HCT116 cells. (A and B) Effects of uc.338 alteration on the cell viability of CRC cells were detected by CCK- 8 assay. (C and D) Representative profiles of EdU cell growth in LoVo and HCT116 cells after uc.338 transfection compared with the negative control (NC) groups. " $\mathrm{P}<0.05$; data are expressed as the mean $\pm \mathrm{SD}$. CRC, colorectal cancer.

antibodies at $4^{\circ} \mathrm{C}$ overnight. After washing in TBST three times (10 min each), the membranes were incubated with secondary antibodies (anti-rabbit or anti-mouse) at room temperature for $2 \mathrm{~h}$. Immunoreactive protein bands were visualized using ECL Plus (Millipore, Billerica, MA, USA) with a Bio-Imaging System. The specific primary antibodies recognized the following proteins: p-PI3K (diluted 1:1,000; cat. no. ab182651; Abcam), PI3K (diluted 1:1,000; cat. no. ab86714; Abcam), p-AKT (diluted 1:500; cat. no. ab38449; Abcam), AKT (diluted 1:500; cat. no. ab8805; Abcam), p21 (diluted 1:5,000; cat. no. ab109520; Abcam) and cyclin D1 (diluted 1:10,000; cat. no. ab134175; Abcam). GAPDH (diluted 1:5,000; cat. no. ab8245; Abcam) was used as an internal control. The secondary antibody was horseradish peroxidase (HRP)-goat anti-rabbit (diluted 1:5,000; cat. no. GAB007; Hangzhou Multi Sciences Biotech, Co., Ltd., Hangzhou, China) or anti-mouse (diluted 1:5,000; cat. no. GAM007; Hangzhou Multi Sciences Biotech, Co., Ltd., Hangzhou, China) IgG.

Statistical analysis. SPSS (version 15.0) (SPSS, Inc., Chicago, IL, USA) and Graphpad Prism5 (GraphPad Software, Inc., La Jolla, CA, USA) were used for the statistical analysis. Data were derived from at least three repeated experiments. The statistical analyses were performed using t-tests, Pearson's $\chi^{2}$ tests and ANOVA. Differences were considered to be statistically significant at $\mathrm{P} \leq 0.05$. The Kaplan-Meier method was used to perform the cumulative survival analysis.

\section{Results}

uc.338 is upregulated in CRC tissues. To reveal the role of uc.338 in CRC progression, we first detected its expression 
A

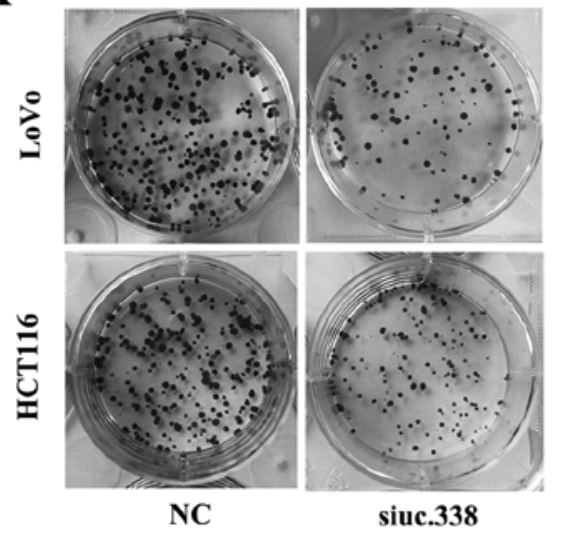

C

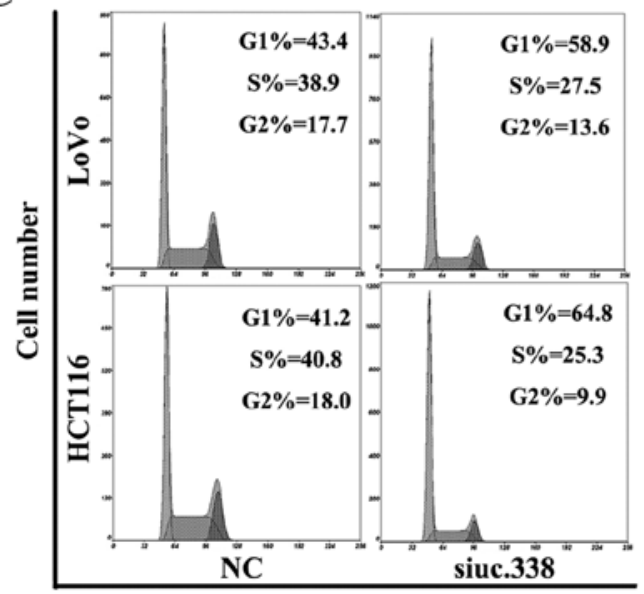

DNA content
B

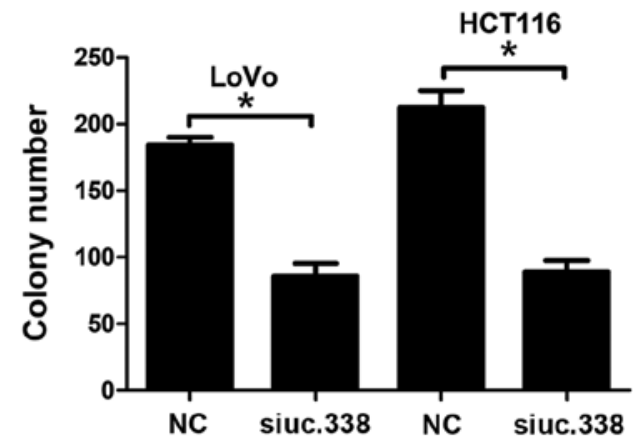

D

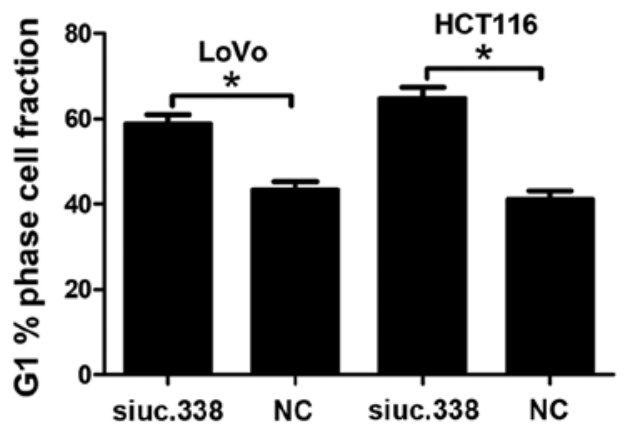

Figure 3. uc. 338 promotes plate colony formation and cell cycle G1/S transition. (A) Effects of uc.338 alteration on plate colony formation of CRC cells. (B) The quantitative statistics of the plate colony formation. (C) Effects of uc.338 on cell cycle distribution in LoVo and HCT116 cells. (D) The quantitative statistics of cell numbers in the G1 phase. ${ }^{*} \mathrm{P}<0.05$; data are expressed as the mean $\pm \mathrm{SD}$. CRC, colorectal cancer.

level in 100 pairs of CRC tissues and adjacent normal tissues using qPCR. The results showed that uc.338 expression was significantly upregulated in CRC tissues compared with that in normal tissues (Fig. 1A). According to the expression level of uc.338 in CRC tissues, we further verified that higher uc.338 expression predicted a larger tumor size, deeper tumor invasion, and increased lymph node metastasis $(\mathrm{P}<0.05$, Table I). However, we did not find any association between uc.338 expression and other clinical features, including sex, age, tumor stage, liver metastasis, location and CEA. We then examined whether the expression level of uc.338 could predict the overall survival (OS) of patients diagnosed with CRC. Using Kaplan-Meier curves, we found that higher uc.338 expression predicted poorer overall survival (OS) in patients with CRC (Fig. 1B). The P-value was $0.012(\mathrm{P}<0.05)$.

Uc.338 is upregulated in CRC cell lines and was inhibited by siRNA. To detect whether uc.338 is upregulated in CRC cell lines, we detected the mRNA expression level of uc.338 using qPCR. Compared with that in the human colon epithelial mucosa cell line NCM460, the mRNA expression of uc.338 was upregulated in LoVo, HCT116 and DLD-1 cells (Fig. 1C).
To knockdown uc.338 expression, LoVo and HCT116 cells were transfected with a siRNA designed to inhibit uc.338 expression (siuc.338). The result revealed that the mRNA expression of uc.338 was significantly inhibited by siuc.338 in both LoVo and HCT116 cells (Fig. 1D and E). The interference efficiency of siuc.338 was 71 and $79 \%$ in LoVo and HCT116 cells, respectively $(\mathrm{P}<0.05)$.

Knockdown of uc.338 inhibits cell proliferation in LoVo and HCT116 cells. To detect the effect of uc.338 on cell proliferation, we performed a CCK-8 assay, an EdU assay, and a plate colony formation assay in succession. In the CCK-8 assay, we found that the viability of LoVo and HCT116 cells was significantly decreased after knockdown of uc.338 (Fig. 2A and B). On the fourth day, the viability of LoVo and HCT116 cells had decreased by 33.9 and $41.1 \%$, respectively $(\mathrm{P}<0.05)$. In the EdU assay, compared with the control cells, we found that the proportion of cells in the DNA replication phase decreased by 19.8 and $21.9 \%$ in the LoVo and HCT116 cells, respectively, after knockdown of uc.338 (Fig. 2C and D), which revealed that knockdown of uc. 338 could inhibit DNA synthesis in CRC cells. In the plate colony formation assay, the result revealed that knockdown of uc.338 significantly 
A

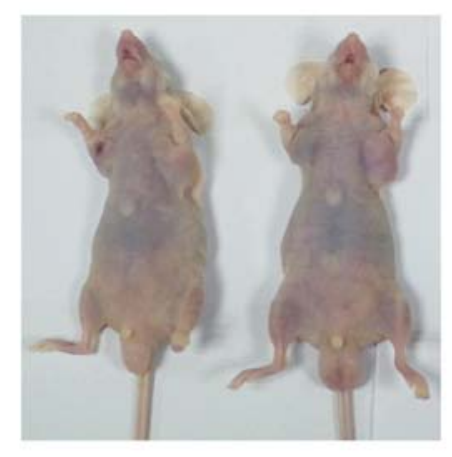

B

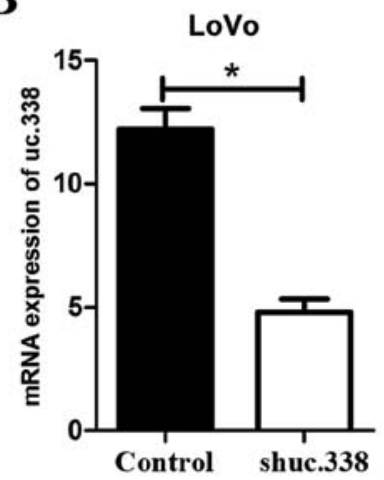

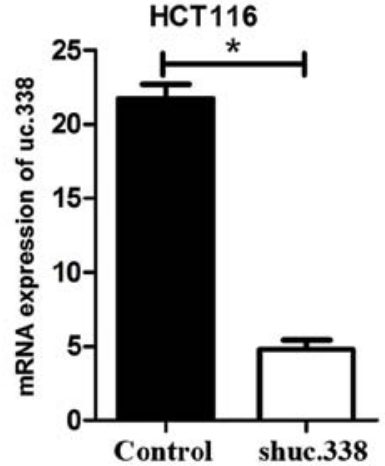

C

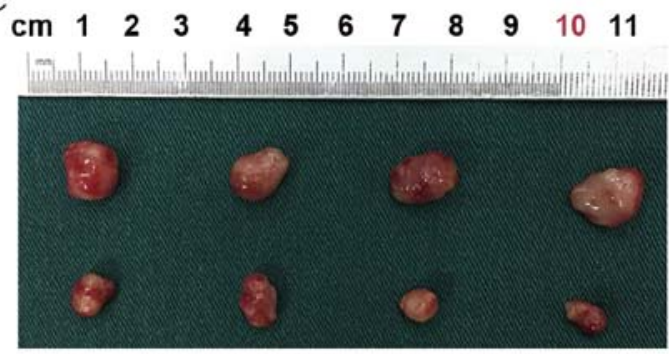

D

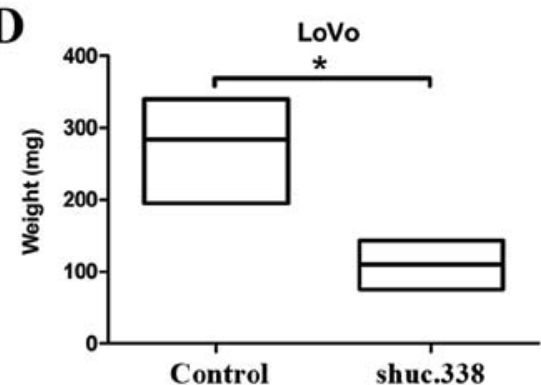

$\begin{array}{llllllllllll}\mathrm{cm} & 1 & 2 & 3 & 4 & 5 & 6 & 7 & 8 & 9 & 10 & 11\end{array}$

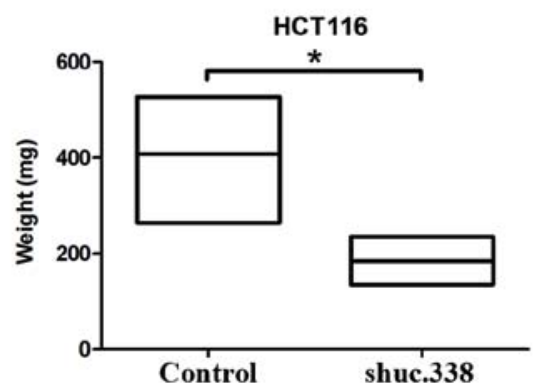

Figure 4. uc.338 promotes tumor growth in nude mice. (A) Nude mice after tumor implantation 4 weeks later in the shuc.338 group (left armpit) and control group (right armpit). Left mouse was injected with LoVo cells and the right was injected with HCT116 cells. (B) Relative mRNA expression of uc.338 in LoVo and HCT116 cells following shuc. 338 transfection. (C and D) The size and weight of tumors formed by LoVo and HCT116 cells in the shuc.338 and control groups. ${ }^{*} \mathrm{P}<0.05$; data are expressed as the mean $\pm \mathrm{SD}$.

inhibited the formation of cell colonies in the LoVo and HCT116 cells (Fig. 3A and B). After 7 days, we found that the number of cell colonies decreased by 46 and $42 \%$ in LoVo and HCT116 cells, respectively, after knockdown of uc.338 $(\mathrm{P}<0.05)$. According to the results of three assays, knockdown of uc.338 inhibited the cell proliferation in CRC LoVo and HCT116 cells.

Knockdown of uc. 338 results in cell cycle arrest in G1/Sphases in LoVo and HCT116 cells. To detect the role of uc.338 in the cell cycle of CRC cells, we performed cell cycle analysis using flow cytometry. After knockdown of uc.338, we found that the cell number in the G1 phase increased by 15.5 and $23.6 \%$ in LoVo and HCT116 cells, respectively, whereas the cell number in the $\mathrm{S}$ phase decreased by 11.4 and $15.5 \%$, respectively, in these two cell lines (Fig. 3C and D). Our result indicated that knockdown of uc.338 caused marked cell cycle arrest in the G1/S phases in the LoVo and HCT116 cells.
Knockdown of uc.338 suppresses tumor growth in nude mice. To detect the role of uc.338 in the tumor growth in nude mice, LoVo and HCT116 cells were transfected with uc.338 inhibitor lentivirus (shuc.338) (Fig. 4B). Then, $2 \times 10^{6}$ cells transfected with shuc.338 and a negative control sequence (control) were separately injected subcutaneously into the nude mice (Fig. 4A). After four weeks, we found that the size and weight of the tumors were significantly decreased in the nude mice transfected with the cells with knockdown of uc.338 (Fig. 4C and D). Compared with the tumors from the control groups, the weight of tumors formed by the uc.338-knockdown LoVo and HCT116 cells was decreased by 61.1 and $54.9 \%$ in the shuc.338 groups $(\mathrm{P}<0.05)$. Therefore, knockdown of uc.338 was able to suppress tumor growth in nude mice.

Downregulation of uc.338 inhibits the expression of cyclin $D 1$ and promotes the expression of $p 21$, possibly via the PI3K/AKT pathway. We revealed that uc.338 could cause cell 
A

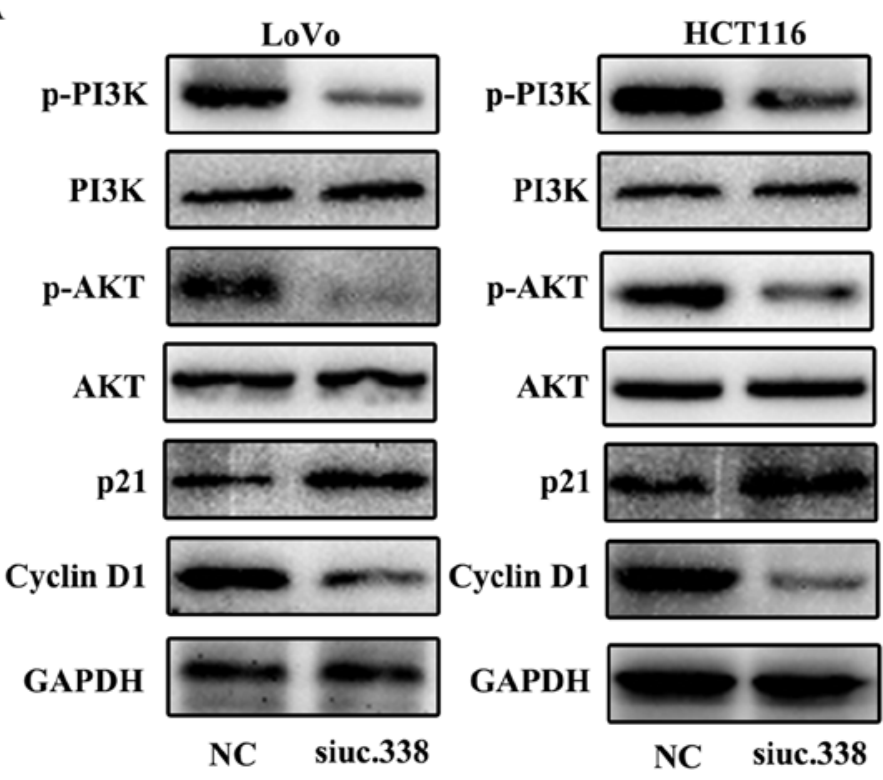

B
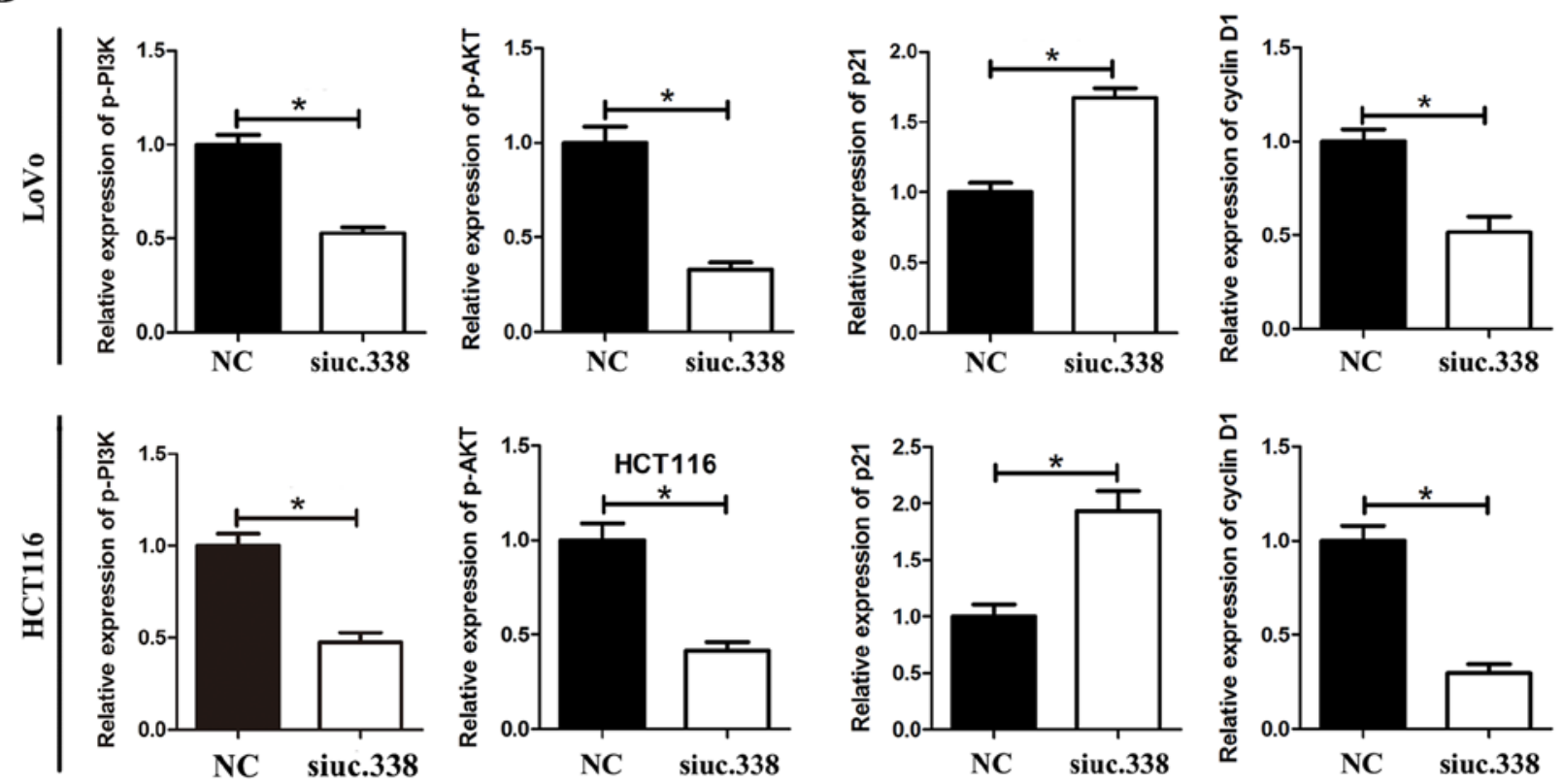

Figure 5. uc.338 possibly promotes p21 downregulation and cyclin D1 upregulation by PI3K/AKT activation. (A) Alterations of p-PI3K, PI3K, p-AKT, AKT, cyclin D1, p21 and GAPDH levels following siuc.338 transfection or in the negative control (NC) in CRC cell lines using western blot analysis. (B) Quantification of the results for p-PI3K, p-AKT, cyclin D1 and p21 as shown in the western blot in A. "P<0.05; data are expressed as the mean \pm SD. CRC, colorectal cancer.

cycle arrest in the G1/S phase in CRC cells. To further detect the potential mechanism of cell cycle arrest, we detected a series of cell cycle-related proteins that could regulate the G1/S phase in LoVo and HCT116 cells. The results showed that the level of cyclin D1 was significantly downregulated and the level of p21 was upregulated by uc. 338 knockdown. Further investigation revealed that the levels of p-PI3K and p-AKT were downregulated by uc. 338 knockdown, whereas no significant change was detected in the levels of total PI3K and AKT (Fig. 5A and B).

To further investigate the role of the PI3K/AKT pathway in uc.338-induced cell proliferation in CRC, a specific inhibitor of PI3K (LY294002) was used. uc.338 was overexpressed in the DLD-1 cell line (Fig. 6A), which expresses a relatively low level of uc.338, and then the cells were treated with LY294002. By western blotting, we found that the PI3K/AKT pathway was significantly inhibited by LY294002 (Fig. 6B and C). The colony formation assay showed that overexpression of uc.338 promoted cell proliferation in DLD-1 cells; however, this promotion of cell proliferation was inhibited by LY294002 (Fig. 6D and E). Taken together, the results demonstrated that knockdown of uc.338 inhibited the expression of cyclin D1 and promoted the expression of p21, possibly via the PI3K/AKT pathway. 


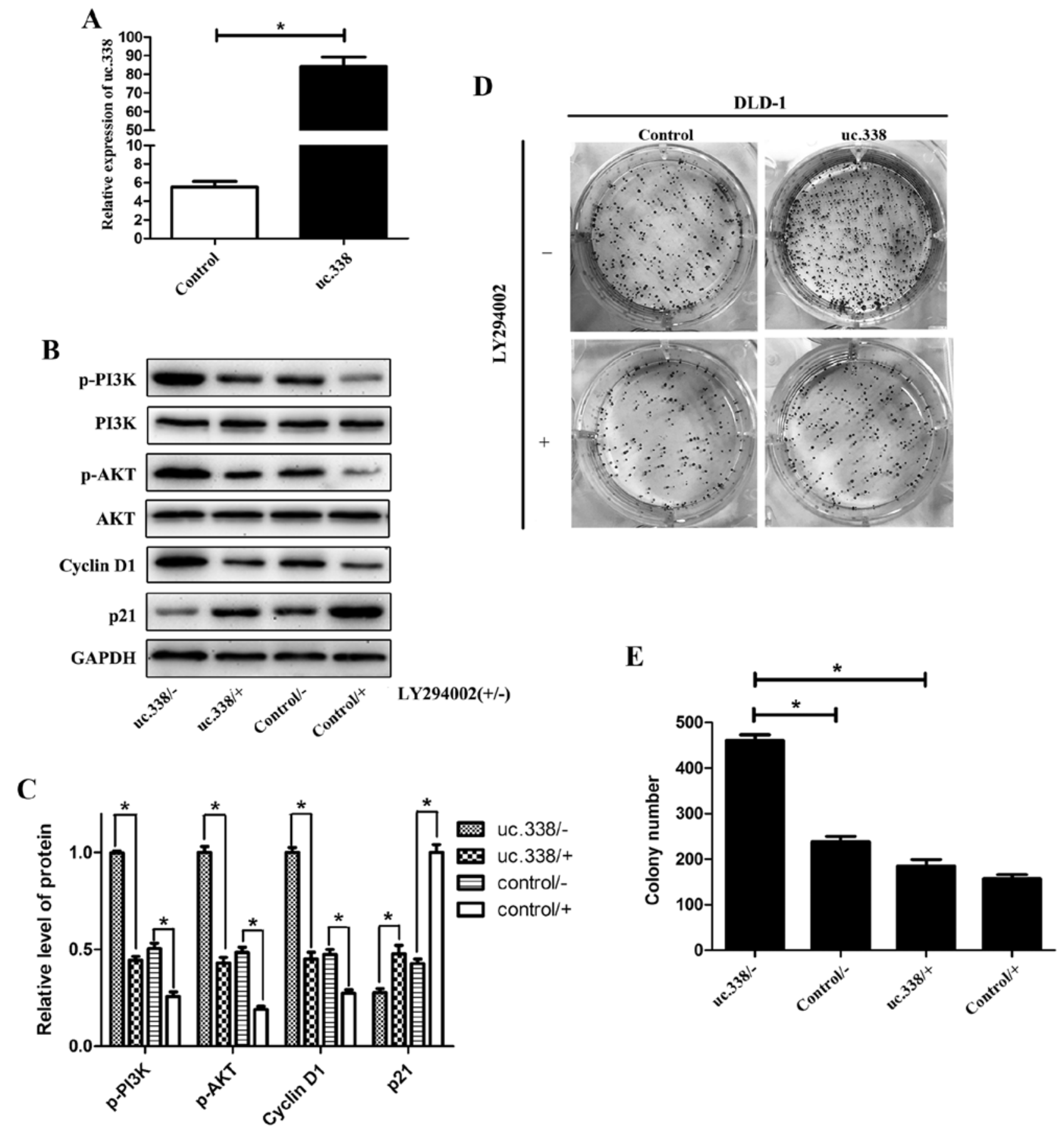

Figure 6. Inhibition of PI3K reduces the function of uc.338-mediated cell proliferation. (A) uc.338 was overexpressed by the plasmid targeted uc.338 in DLD-1 cells. (B and C) The PI3K/AKT pathway was inhibited by LY294002 in DLD-1 cells under different treatments. (D) DLD-1 cells transfected with the plasmid targeted uc.338 and LY294002 or not were utilized for the colony formation assay. (E) The quantitative statistics of the plate colony formation in DLD-1 cells. ${ }^{*} \mathrm{P}<0.05$; data are expressed as the mean $\pm \mathrm{SD}$.

\section{Discussion}

Their high degree of conservation means that ultraconserved regions (UCRs) are considered to have fundamental functional importance for the ontogeny and phylogeny of mammals and other vertebrates (3). Since Calin et al first detected the role of T-UCRs in human cancers (6), increasing evidence has shown that aberrant expression of T-UCRs is involved in tumor pathogenesis. Simultaneously, single nucleotide polymorphisms (SNPs) within UCRs were also verified to be closely related to clinical features in various carcinomas (15-17). These observations revealed the vital importance of T-UCRs in human carcinomas. uc.338 is an exonic type T-UCR that overlaps with the mRNA of a known human protein-coding gene $(6,18)$. Previous studies have shown that uc.338 is involved in the progression of various tumors. For example, Bo et al found that uc.338 promoted HCC cell proliferation and induced cell cycle progression through an association with BMI1 in hepatocellular carcinoma (10). Gao et al found that uc.338 could promote proliferation and metastasis by 
cyclin B1 and EMT activation in lung carcinoma (13). In CRC, Wang et al first detected that uc.338 could promote CRC cell migration and invasion by targeting the TIMPI (14); however, the role of uc.338 in CRC cell proliferation was not completely clear.

In the present study, we first detected that the expression level of uc.338 mRNA was upregulated in CRC tissues. On this basis, we further analyzed the clinical features and prognosis of patients with CRC. The results indicated that a higher expression level of uc.338 mRNA was associated with larger tumors, deeper tumor invasion, increased lymph node metastasis, and poorer prognosis. Our results not only confirmed the results of Wang et al, but also suggested a possible role of uc. 388 in CRC proliferation and prognosis. To further examine the role of uc.338 in promoting cell proliferation, we performed a series of cell proliferation experiments in vivo and in vitro. In vitro, we found that uc.338 was significantly upregulated in CRC cell lines (LoVo, HCT116 and DLD-1) compared with its level in normal colon epithelial cells (NCM460). Further experiments revealed that the cell viability, DNA replication, and colony formation were significantly inhibited by uc.338 knockdown in LoVo and HCT116 cells. In vivo, we transplanted CRC cells into nude mice, which revealed that the speed of tumor growth from cells with uc.338 knockdown was significantly inhibited, which supported our hypothesis that uc.338 could promote cell proliferation in CRC. Flow cytometry showed that the cell cycle in the G1/S phase was arrested by uc.338 inhibition, suggesting that uc.338 could promote cell cycle transition. However, no difference was found in the rate of cell apoptosis in CRC cells, indicating that uc.338 is not involved in cell apoptosis control (data not shown). These results indicated that uc.338 promotes tumor proliferation possibly via cell cycle $\mathrm{G} 1 / \mathrm{S}$ promotion in CRC cells.

Numerous components of the cell cycle kinases or kinase inhibitors are involved in mediating the $\mathrm{G} 1 / \mathrm{S}$ transition $(19,20)$. Protein p21 is generally recognized as a negative regulator in the G1/S transition (21), and downregulation of $\mathrm{p} 21$ is involved in tumor promotion in various cancers $(22,23)$. Conversely, cyclin D1 is widely accepted as an oncogene and has been implicated in many activities, such as cell cycle promotion, chromosomal instability, mitochondrial function, and cellular senescence (24-26). To better understand the mechanisms that promote the G1/S cell cycle transition mediated by uc.338, the expression levels of certain cell cycle-related proteins were determined using western blotting. Our results indicated that arrest of the cell cycle at the G1/S phase in CRC cells might be facilitated by uc.338-mediated p21 upregulation and cyclin D1 downregulation. To further understand the mechanism of uc.338-mediated regulation of p21 and cyclin D1, we detected the protein levels of key members of the PI3K/AKT pathway. The PI3K/AKT pathway is recognized as a classic signaling mediator that modulates cell proliferation and apoptosis, and is involved in the progression of various tumors (26-28). In this process, p21 and cyclin D1 are two common downstream regulatory genes of the PI3K/AKT pathway in a variety of tumors, including CRC $(29,30)$. Western blotting showed that the levels of $\mathrm{p}-\mathrm{PI} 3 \mathrm{~K}$ and p-AKT were significantly decreased by uc.338 knockdown in CRC cells, whereas no significant difference was found in the total PI3K and AKT levels. Moreover, after treatment with a specific inhibitor of PI3K (LY294002), the oncogenic role of uc.338 in cell proliferation was distinctly inhibited. Hence, we hypothesized that uc.338 could promote p21 downregulation and cyclin D1 upregulation, possibly via PI3K/AKT pathway activation.

p53, usually called as 'guardian of the genome', is an important tumor-suppressor gene that controls response to oncogene activation such as cell cycle arrest, apoptosis, and senescence. In the mammalian genome, p53 is also the most frequently mutated gene in human cancer (31-33). It has been reported that elevated PI3K/Akt activity is observed in cells harboring high levels of p53 mutant protein $(34,35)$, and p53 could bind to $\mathrm{p} 21$ to promote cell death or replicative senescence $(36,37)$. To date, no direct evidence has shown that uc. 338 is related to p53 status. To further investigate whether uc.338 is associated with p53, we detected the mRNA expression of p53 by uc.338 knockdown, but our results showed no significant relationship between uc.338 andp53 expression. Even so, it remains unclear whether uc. 338 is related to the p53 mutation or in other states, and a more in-depth study on the relationship between uc.338 and p53 should be performed in our next study. Meanwhile, the aberrant regulation of T-UCR expression consistently plays a role in cancer by altered interactions with miRNAs (18). It is uncertain whether uc.338 interacts with certain miRNAs, and we cannot rule out the possibility that other signaling pathways might also be affected by uc.338 in regards to CRC proliferation. Furthermore, the exact association between uc.338, p21, cyclin D1 and the PI3K/AKT pathway requires further investigation.

In conclusion, we demonstrated that uc. 338 was significantly upregulated in CRC tissues. We proposed that uc.338 expression is associated with tumor size, invasion depth, and prognosis in patients with CRC. Further study provided evidence supporting our hypothesis that uc. 338 promotes CRC cell proliferation by facilitating cell cycle G1/S transition. Finally, we indicated that uc.338 might target p21 and cyclin D1 to promote cell cycle G1/S transition, possibly by activation of the PI3K/Akt pathway.

\section{Acknowledgments}

We would like to thank Dr Hao Fan for the excellent technical assistance. We also would like to thank Dr Yuanguangyan Zhang for the great statistical guidance.

\section{Funding}

The present study was supported by the Jiangsu Key Medical Discipline (General Surgery) (grant no. ZDXKA2016005).

\section{Availability of data and materials}

The datasets used during the present study are available from the corresponding author upon reasonable request.

\section{Authors' contributions}

YS, ZF and YZ contributed to the design of this study. ShijiaW, WQ and ZZ contributed to the experimental work. YZ, BJ and 
SenW contributed to the data collection and analysis. YZ, QW and DJ contributed to the interpretation of data and drafting the manuscript. All authors read and approved the manuscript and agree to be accountable for all aspects of the research in ensuring that the accuracy or integrity of any part of the work are appropriately investigated and resolved.

\section{Ethics approval and consent to participate}

The present study using human tissues was approved by the Ethics Committee of the First Affiliated Hospital of Nanjing Medical University. Our animal experiments were approved by the Animal Ethics Committee of Nanjing Medical University (NJMU).

\section{Patient consent for publication}

Not applicable.

\section{Competing interests}

The authors declare that they have no competing interests.

\section{References}

1. Torre LA, Bray F, Siegel RL, Ferlay J, Lortet-Tieulent J and Jemal A: Global cancer statistics, 2012. CA Cancer J Clin 65: 87-108, 2015.

2. Chen W, Zheng R, Baade PD, Zhang S, Zeng H, Bray F, Jemal A, Yu XQ and He J: Cancer statistics in china, 2015. CA Cancer J Clin 66: 115-132, 2016

3. Bejerano G, Pheasant M, Makunin I, Stephen S, Kent WJ, Mattick JS and Haussler D: Ultraconserved elements in the human genome. Science 304: 1321-1325, 2004.

4. Kung JT, Colognori D and Lee JT: Long noncoding RNAs: Past, present, and future. Genetics 193: 651-669, 2013.

5. Mestdagh P, Fredlund E, Pattyn F, Rihani A, Van Maerken T, Vermeulen J, Kumps C, Menten B, De Preter K, Schramm A, et al: An integrative genomics screen uncovers ncRNA T-UCR functions in neuroblastoma tumours. Oncogene 29: 3583-3592, 2010.

6. Calin GA, Liu CG, Ferracin M, Hyslop T, Spizzo R, Sevignani C, Fabbri M, Cimmino A, Lee EJ, Wojcik SE, et al: Ultraconserved regions encoding ncRNAs are altered in human leukemias and carcinomas. Cancer Cell 12: 215-229, 2007.

7. Sana J, Hankeova S, Svoboda M, Kiss I, Vyzula R and Slaby O Expression levels of transcribed ultraconserved regions uc.73 and uc.388 are altered in colorectal cancer. Oncology 82: 114-118, 2012.

8. Hudson RS, Yi M, Volfovsky N, Prueitt RL, Esposito D, Volinia S, Liu CG, Schetter AJ, Van Roosbroeck K, Stephens RM, et al: Transcription signatures encoded by ultraconserved genomic regions in human prostate cancer. Mol Cancer 12: 13, 2013.

9. Braconi C, Valeri N, Kogure T, Gasparini P, Huang N, Nuovo GJ, Terracciano L, Croce CM and Patel T: Expression and functional role of a transcribed noncoding RNA with an ultraconserved element in hepatocellular carcinoma. Proc Natl Acad Sci USA 108: 786-791, 2011.

10. Bo C, Li N, Li X, Liang X and An Y: Long noncoding RNA uc.338 promotes cell proliferation through association with BMI1 in hepatocellular carcinoma. Hum Cell 29: 141-147, 2016.

11. Jin W, Chen L, Cai X, Zhang Y, Zhang J, Ma D, Cai X, Fu T, Yu Z, Yu F and Chen G: Long non-coding RNA TUC338 is functionally involved in sorafenib-sensitized hepatocarcinoma cells by targeting RASAL1. Oncol Rep 37: 273-280, 2017.

12. Li Q, Shen F and Wang C: TUC338 promotes cell migration and invasion by targeting TIMP1 in cervical cancer. Oncol Lett 13 : 4526-4532, 2017

13. Gao X, Gao X, Li C, Zhang Y and Gao L: Knockdown of long noncoding RNA uc.338 by siRNA inhibits cellular migration and invasion in human lung cancer cells. Oncol Res 24: 337-343, 2016.
14. Wang C, Wang Z, Zhou J, Liu S, Wu C, Huang C and Ding Y: TUC.338 promotes invasion and metastasis in colorectal cancer. Int J Cancer 140: 1457-1464, 2017.

15. Catucci I, Verderio P, Pizzamiglio S, Manoukian S, Peissel B, Barile M, Tizzoni L, Bernard L, Ravagnani F, Galastri L, et al: SNPs in ultraconserved elements and familial breast cancer risk. Carcinogenesis 30: 544-545; author reply 546, 2009.

16. Bao BY, Lin VC, Yu CC, Yin HL, Chang TY, Lu TL, Lee HZ, Pao JB, Huang CY and Huang SP: Genetic variants in ultraconserved regions associate with prostate cancer recurrence and survival. Sci Rep 6: 22124, 2016.

17. Shen H, Lu C, Jiang Y, Tang J, Chen W, Zhang H, Zhang Q, Wang J, Liang J, Hu Z and Shen H: Genetic variants in ultraconserved elements and risk of breast cancer in chinese population. Breast Cancer Res Treat 128: 855-861, 2011.

18. Peng JC, Shen J and Ran ZH: Transcribed ultraconserved region in human cancers. RNA Biol 10: 1771-1777, 2013.

19. Motokura T and Arnold A: Cyclin D and oncogenesis. Curr Opin Genet Dev 3: 5-10, 1993.

20. Pines J: Cyclins: Wheels within wheels. Cell Growth Differ 2: 305-310, 1991.

21. Niculescu AB III, Chen X, Smeets M, Hengst L, Prives C and Reed SI: Effects of p21(Cip1/Waf1) at both the G1/S and the G2/M cell cycle transitions: $\mathrm{PRb}$ is a critical determinant in blocking DNA replication and in preventing endoreduplication. Mol Cell Biol 18: 629-643, 1998.

22. Nie FQ, Sun M, Yang JS, Xie M, Xu TP, Xia R, Liu YW, Liu XH, Zhang EB, Lu KH and Shu YQ: Long noncoding RNA ANRIL promotes non-small cell lung cancer cell proliferation and inhibits apoptosis by silencing KLF2 and P21 expression. Mol Cancer Ther 14: 268-277, 2015.

23. Abbas T and Dutta A: p21 in cancer: Intricate networks and multiple activities. Nat Rev Cancer 9: 400-414, 2009.

24. Casimiro MC and Pestell RG: Cyclin d1 induces chromosomal instability. Oncotarget 3: 224-225, 2012.

25. Leontieva OV, Lenzo F, Demidenko ZN and Blagosklonny MV: Hyper-mitogenic drive coexists with mitotic incompetence in senescent cells. Cell Cycle 11: 4642-4649, 2012.

26. Wang C, Li Z, Lu Y, Du R, Katiyar S, Yang J, Fu M, Leader JE, Quong A, Novikoff PM and Pestell RG: Cyclin D1 repression of nuclear respiratory factor 1 integrates nuclear DNA synthesis and mitochondrial function. Proc Natl Acad Sci USA 103: 11567-11572, 2006.

27. Chen J, Zhao KN, Li R, Shao R and Chen C: Activation of $\mathrm{PI} 3 \mathrm{~K} / \mathrm{Akt} / \mathrm{mTOR}$ pathway and dual inhibitors of PI3K and mTOR in endometrial cancer. Curr Med Chem 21: 3070-3080, 2014.

28. Lee JJ, Loh K and Yap YS: PI3K/Akt/mTOR inhibitors in breast cancer. Cancer Biol Med 12: 342-354, 2015.

29. Wang L, Cao XX, Chen Q, Zhu TF, Zhu HG and Zheng L: DIXDC1 targets $\mathrm{p} 21$ and cyclin D1 via PI3K pathway activation to promote colon cancer cell proliferation. Cancer Sci 100: 1801-1808, 2009.

30. Yan L and Shi G: Effect of IFN- $\alpha$ on hepatic cancer SMCC-7721 cell via PI3K/Akt signaling pathway and related mechanism research. Zhonghua Yi Xue Za Zhi 95: 2960-2963, 2015 (In Chinese).

31. Liu J, Zhang C, Hu W and Feng Z: Tumor suppressor p53 and its mutants in cancer metabolism. Cancer Lett 356: 197-203, 2015.

32. Liu J, Zhang C and Feng Z: Tumor suppressor p53 and its gain-of-function mutants in cancer. Acta Biochim Biophys Sin (Shanghai) 46: 170-179, 2014.

33. Duffy MJ, Synnott NC, McGowan PM, Crown J, O'Connor D and Gallagher WM: p53 as a target for the treatment of cancer. Cancer Treat Rev 40: 1153-1160, 2014.

34. Muller PA, Caswell PT, Doyle B, Iwanicki MP, Tan EH, Karim S, Lukashchuk N, Gillespie DA, Ludwig RL, Gosselin P, et al: Mutant p53 drives invasion by promoting integrin recycling. Cell 139: 1327-1341, 2009.

35. Hanel W, Marchenko N, Xu S, Yu SX, Weng W and Moll U: Two hot spot mutant p53 mouse models display differential gain of function in tumorigenesis. Cell Death Differ 20: 898-909, 2013.

36. Riley T, Sontag E, Chen P and Levine A: Transcriptional control of human p53-regulated genes. Nat Rev Mol Cell Biol 9: 402-412, 2008.

37. Haupt S, Berger M, Goldberg Z and Haupt Y: Apoptosis-the p53 network. J Cell Sci 116: 4077-4085, 2003. 